\title{
Ergonomic factors that cause the presence of pain muscle in students of dentistry
}

\author{
Antonio-José Diaz-Caballero ${ }^{1}$, Isabel-Patricia Gómez-Palencia ${ }^{2}$, Shyrley Díaz-Cárdenas ${ }^{3}$
}

\footnotetext{
${ }^{1}$ Dentist University of Cartagena. Specialist in Periodontics Universidad Javeriana. Master of Education North University. Candidate of Biomedical Sciences University of Cartagena. Titular Professor University of Cartagena

${ }^{2}$ Nurse University of Cartagena. Occupational Health Specialist. Professor University of Cartagena

${ }^{3}$ Dentist University of Cartagena. Health Management Specialist Technologic University of Bolívar. Family Health Specialist North University. Candidate of Master Public Health National University. Professor University of Cartagena
}

Correspondence:

Health Campus,

Cartagena de Indias,

Bolivar, Colombia,

antoniodiazc@yahoo.com

Received: $12 / 08 / 2009$

Accepted: 25/03/2010

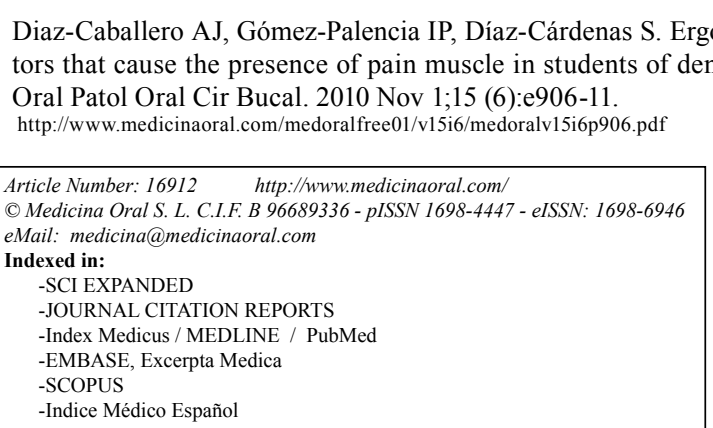

\begin{abstract}
Objective: To identify the ergonomic factors and the presence of muscular pain in dental students of VIII, IX, $\mathrm{X}$ semesters presently practicing at the clinics of the College of Dentistry of university of Cartagena, Colombia, South America.

Materials and methods: This is a descriptive study carried out in dental students of the VIII, IX, and X semesters which were undergoing clinical practice at the College of Dentistry of University of Cartagena. A convenience sample of 83 students who met the inclusion criteria was taken and those who agreed to participate signed an informed consent. Data collection was obtained by means of a structured questionnaire for ergonomic factors and the presence of pain was identified palpating the muscles object of the study by a physical therapist. The variables evaluated were: presence of pain, affected muscles, affected zones, gender, postures and work environment.

Results: The adoption of inadequate postures such as exaggerated flexions or cervical torsions could generate a higher frequency of muscular pain in dental students. The approximating muscle of the thumb showed $11 \%$ frequency of pain. The presence of muscular pain was higher for the female group participating in this study.

Conclusions: Dentists are prone, since the beginning of their clinical practice as students, to lesions of the skeletal muscle system due to the clinical exercise of the profession, being the most common, muscle pain in the back, neck, shoulders and hands. This would imply initiating an occupational health program promoting healthy lifestyles in their academic environment and their future professional life, at the beginning of their clinical practice in dental school.
\end{abstract}

Key words: Muscular pain, dental students, ergonomics, postures. 


\section{Introduction}

Dentistry is a profession that generally produces muscular pain and soreness, they are usually harmless and slow to appear, consequently, the symptoms are usually ignored until they become chronic and permanent lesions are present (1).

Occupational health is defined by WHO as a multidisciplinary activity which promotes and protects workers health. This discipline seeks to control accidents and diseases through reduction of risks. Dentistry, as a form of work, is not exempt of risks. Generically, the possible risk factors can be classified as: Biomechanical, ergonomic and work factors. In dentistry, factors such as physical conditions of the environment, adequate equipment design with proper anthropometric requirements, and avoidance of unhealthy postures can be evaluated from the ergonomic point of view (2). Regarding ergonomics, this discipline proposes the provision of working conditions which promote workers welfare and facilitate the performance of labor, it also includes the design of work spaces, equipment, the environment and the process to adequate them to the physical and psychological characteristics of the human being (3). It is very important to maintain an adequate work posture and that the instruments and furniture that the dentist is working with, have adequate working characteristics (4). When practicing, dentists sometimes are forced to adopt unhealthy postures which depend mainly on factors related to the working conditions (5). Also, they are exposed to biomechanical risk factors, which indicate that work forced postures, would imply more risk of soreness and presence of skeletal-muscle lesions. These lesions could begin to appear at the beginning of their clinical practice as students, by acquiring inadequate postures and working habits that will accompany them for the rest of their professional life (6), acquiring an unhealthy lifestyle in their work environment.

Several studies have addressed the relationship between inadequate postures while practicing dentistry and the appearance of pain or muscular lesions. Lake in 1995 implicates several mechanisms in the generation of pains and soreness in dentists, such as: a) elevated work area with permanent static positions of more than 30 degrees, which would produce a reduction of blood flow in the supra spine tendon and would also originate high muscle tension on the trapezoids (1). b) Lack of support of the forearms during repetitive holding of instruments which would compromise different body segments such as spine, shoulder, and wrists $(1,7)$. The precision required forces the dentist to maintain forced wrist postures which might produce tendinitis or carpal tunnel syndrome. (8). c) The handling of vibrating instruments is associated with specific lesions such as nerve trapping, early arthrosis and even, with Raynaud syndrome $(7,8)$. d) Forced cervical static postures. In order to obtain a good vision of the mouth, dentists frequently adopt cervical torsions and flexions which end up in pain. $(8,9)$. e) Poor posture when seating. The flexion of the lumbar spine, when seating forward, produces marked pressure increments between the interdiscal spaces $(8,9)$. f) Lighting at the work place: the lack or excess of light can generate myopia and irreversible retinal lesions, among others (10). g) Temperature, ventilation and humidity at the work place. If the temperature is high and the air is saturated with humidity, there is exhaustion, increased body temperature and, respiratory and circulatory disorders (11). h) Noise at the work place. Intermittent and continuous noise produced by high and low speed instruments is, according to many professionals, the biggest responsible for auditive alterations as years go by $(12,13)$. i) Equipment. Present dental chairs allow adaptation of the patient's position in height, inclination of the torso, flexion or hyper extension of the head of the patient. On the other side, both the dentist's chair as well as the dental assistant's must permit height regulation; allow controlling the height of the back of the chair and even, regulate the lumbar-dorsal support in an anterior-posterior position and, the padding must be thin and permanent (14).

Occupational Health Programs are not being carried out in a satisfactory manner nor is the adequate training of these activities being promoted. This is why, a study carried out in Italy, suggests how muscular skeletal disorders can be minimized through prevention, ergonomic strategies and specific therapeutic programs. Prevention includes early identification of symptoms, analysis of the activity and work posture and, equipment evaluation such as position of the dental unit, hand instruments, operator and patient's chair as well as the lighting conditions (15).

Valachi et al. in 2003 also found a positive association between presence of pain and specific forced postures: torsion of the trunk, moving the shoulders towards the side, elevating the elbows, operating light too far away from the line of vision when working on the maxillary arch, working with the hands close to the patient's face and working for long periods of time.

There are other factors related with the appearance of pain and muscular lesions such as gender, being higher in female dentists (16), and age, although this is controversial. Although undoubtedly the age of the dentist is closely related with how many years the dentist has been practicing, some studies found that this condition can be evaluated as an independent variable. (17). For some authors, back and cervical pain remain stable with age. Other authors consider that muscular-skeletal disorders are at their peak around the 6th decade, meaning, they increase progressively with age. A third group considers that the disorders are higher in younger professionals. They believe that this could be due to the 
healthy worker effect, (the sick ones retired early) or to different forms of work in function of age. There are studies reporting more neck pain in dentists older than 30 years of age, than in younger professionals (17).

In order to study these topics, professional dentists usually are the population to be evaluated, but few studies have been done with dental students, which is the population that, during their course of study, acquires habits and practices that could become generators of health protection. Within these habits we find acquired inadequate postures in their place of practice. For these reasons it becomes necessary to find out if there is a presence or absence of muscular pain and the possible ergonomic factors associated in dental students, in order to implement preventive and corrective measures arising from the college institutions, generating and promoting healthy lifestyles during their professional practice.

\section{Materials and Methods}

A transversal descriptive study was carried out. The initial sample consisted of a total of 110 students, from the VIII, IX and X semesters which were going through their clinical practice at the College of Dentistry, University of Cartagena. From the initial sample, 7 students withdrew from the study, 12 refused to participate and 8 of them were discarded for not meeting the inclusion criteria, for a total sample of 83 students who met the following inclusion criteria: Students from the VIII, IX, X semesters going through their clinical practice at the College of Dentistry of the University of Cartagena who wanted to participate in the study (informed consent) and, as exclusion criteria, the following were established: students suffering from previously diagnosed scoliosis, chronic lumbar pain, those who also perform other work activities requiring forced postures, who have been subjected to disc hernia surgery, history of fractures in the trunk or lower members, history of muscular tear of lower members, students who practiced sports at least three times a week.

The variables included in the study were 1) presence of pain; 2) muscles affected (deltoids, trapezoid (upper, medium, lower fibers), sternocleidomastoid, wide dorsals, obliques, rectoabdominal, biceps, lumbar, paravertebrals, rhomboids, palmar major, palmar minor, pronators, supinators, long flexor of the thumb, short flexor of the thumb and, approximating of the thumb. (30);3) affected zones (lumbar zone, dorsal zone, cervical zone, neck, shoulders, forearm, arm, arm, hand), 4) gender, 5) posture 6) working place. Posture analysis was evaluated using the EWA (Ergonomic Workplace Analysis), this is an instrument which allows analysis of the situation at the workplace and is based on work physiology, occupational biomechanics, information psychology, industrial hygiene and the socio-technical model of organization of the work (18).
The instrument was designed and calibrated with theoretical parameters and with the advice of occupational health and physical therapist specialists with suggested criteria validated by their experience. Then, the examiners were standardized with respect to the filling of the questionnaire to carry out the concordance tests between observations using a Kappa Cohen analysis, with an agreement degree of 0.81 with respect to a golden standard (EWA, instrument of ergonomic analysis of the workplace). A pilot study with the participation of 15 students from the VIII, IX and X semesters and who met the inclusion criteria was done, they were evaluated by a physical therapist before filling out the questionnaire, this was done in order to unify criteria, provide better validation of the instrument and reduce bias; it was then decided to make some changes in the order of the procedure and modify some points in the questionnaire. The order in which the instrument was applied had the purpose of avoiding false positives, since the subjects participating in the study could be predisposed for the presence of pain; therefore, muscular evaluation was done before filling out the questionnaire. Data collection was carried out in two phases: for the first phase, the self processing instrument was used, this consisted of a structured questionnaire with multiple choice closed questions with only one answer, and others with multiple choice and multiple answers (See annexed questionnaire). After the subjects answered the questionnaire, they moved to a specially prepared room in an enclosed space with gurney and a specialist in physical therapy for the second phase, which consisted of muscular palpation by the physical therapist and where the subjects informed about the presence or absence of muscular pain at the moment the professional palpated the muscles being evaluated in this study.

The presentation of results was done by means of frequency tables to include the variables in an individual manner; for the determination of the crossing of variables, double entry or association tables were done and, the frequency tables were schematized with a simple bar graph or pie graph.

A descriptive analysis was done using frequencies and percentages for each of the variables studied. The only quantitative variable is age, but this one did not show any variation among the participants in the study and it was not evaluated.

A Chi square test with $95 \%$ of probability and $0.5 \%$ margin error was done to establish differences when relating two qualitative variables and, to find out if there was statistical significance or were due to chance.

Considering that this was an intervention study with minimum risk, referenced by the technical, scientific and administrative norm for health research (Resolution 008430 of 1993, Ministry of Health, Republic of Colombia), an informed consent was designed for all the participants. 


\begin{abstract}
ANNEX
Questionnaire used for this study ergonomic factors associated with muscular pain in dental students of the viii, ix, $\mathrm{x}$ semester going trough clinical practice at the College of Dentistry of University of Cartagena

OBJECTIVE: To determine the ergonomic factors which produce muscular pain in dental students of the VIII, IX, $\mathrm{X}$ semester going through clinical practice at the college of dentistry of university of Cartagena.
\end{abstract}

Number of questionnaire:

Name:

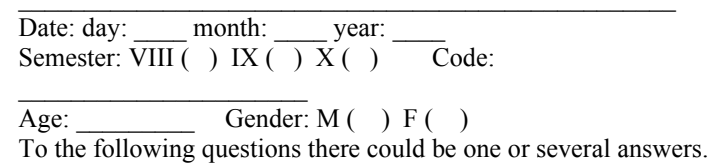

1. Do you have muscular pain due to dental practice

Yes ( ) No ( )

2. Are you familiar with the ergonomic posture to perform clinical procedures in your dental practice

Yes ( ) No ( )

3. Do you experience pain during or after using vibrating instruments ( high speed handpiece, micro motor, contrangle)

Yes ( ) No ( )

4. Which activities of your clinical practice produce muscular pain? Mark the main activities

Surgery ( ) Endodontics ( ) Periodontics ( ) Restorative ( )

Mark in which zone do you you feel pain

5. Lumbar zone (lower back)

Yes ( ) No ( )

6. Dorsal zone (mid back)

Yes ( ) No ( )

7. Cervical zone (upper back)

Yes ( ) No ( )

8. Neck

Yes ( ) No ( )

9. Shoulders

Yes ( ) No ( )

10. Forearm

Yes ( ) No ( )

11. Arm

Yes ( ) No ( )

12. Wrist

Yes ( ) No ( )

13. Hand

Yes ( ) No ( )

14. Are you able to change your work posture, seating or standing, during your practice

Yes ( ) No ( )

15. Do you frequently change positions during your clinical practice

Yes ( ) No ( )

16. After finishing clinical practice, do you perform stretching exercises

Yes ( ) No ( )

17. Are the instruments within hand reach without making strenuous movements?

Yes ( ) No ( )

18. Do you perform torsions or cervical flexions to improve vision when working in the oral cavity

Yes ( ) No ( )
19. Do you cross your legs when working

Yes ( ) No ( )

20. Do you work with your legs slightly separated

Yes ( ) No ( )

21. Do you support yourself on your feet's sole when working

Yes ( ) No ( )

22. When you work, do you put your elbows and arms on the ribs side (ribs)

Yes ( ) No ( )

23. Do you keep your arms at sternum height when working

Yes ( ) No ( )

24. At what distance do you put your elbows in relation to the patient's mouth

a. At the same height

b. Approx $5 \mathrm{~cm}$ above

c. Approx $5 \mathrm{~cm}$ below

d. Approx $10 \mathrm{~cm}$ above

e. Approx $10 \mathrm{~cm}$ below

25. Your position on the stool is

POSITION IN THE STOOL

Forward In the middle backwards

26. Your support on the back of the stool is:

27. The curvature of your spine when working is:

28. The inclination of the chest when working is:

29. Your lateral inclination is:

30. The inclination of your neck is :

31. The position of your forearms is:

32. Do you support your forearms when making repetitive movements

Yes ( ) No ( )

33. Do you have enough space in your workplace

Yes ( ) No ( )

34. Is the work stool comfortable

Yes ( ) No ( )

35. Do you consider that the dental stool you are using for your clinical practice can be ergonomically regulated for the individual needs (vertical height of the body, horizontal regulation of the back, vertical regulation of the back).

Yes ( ) No ( )

36. When initiating your practice, do you position your chair to maintain an ergonomic posture

Si ( ) No ( )

37. D you consider that you have sufficient light at your workplace

Yes ( ) No ( )

38. Do you think that the work instruments are in optimal conditions so you don't have to do extra work (curettes, files, burs, elevators, etc)

Yes ( ) No ( ) 


\section{Results}

- Gender: $63 \%$ of the sample corresponded to females and $37 \%$ were males.

- Presence of pain: $80 \%$ of the students reported muscular pain due to the clinical practice; the clinical areas where more pain was found were surgery and periodontics.

- Areas affected with pain: $15 \%$ of the students reported pain in the neck and lumbar zone, $13 \%$ reported pain in the cervical, dorsal and wrist zones followed by $6 \%$ in the forearm and arm.

- Muscles affected: they were divided in right and left resulting in a higher frequency of pain in the approximating thumb in both sides; the long flexor of the thumb and the deltoid presented less pain.

- Postures adopted: $89 \%$ of the participants varied the working posture during clinical practice, $67 \%$ frequently change positions and only $13 \%$ did stretching exercises, $55 \%$ of the students reported that the instruments were at hands reach without making unnecessary uncomfortable movements.

It was also found that a high percentage of students usually work with their legs slightly separated, with the sole of the feet and the spine resting on the back of the stool when working.

Additionally, a large majority performed torsions or cervical flexions to improve vision when working, adopting an inclined posture of the neck and maintaining a seating position in the middle of the stool. On the other hand, $60 \%$ of the students do not place their hands at the height of the sternum and perform lumbar inclination of the spine.

- Condition of the workplace: $61 \%$ of the students consider that they have sufficient space in their workplace but they consider that the work stool is uncomfortable.

- Relation among variables: the pain variable was related with the following variables: semester, gender, knowledge of the clinical posture of the dentist, torsion or cervical flexions to improve vision, position of the stool, and curvature of the spine when working. $\mathrm{P}<0,05$ values were found for the Chi square test when relating presence of pain and torsions or cervical flexions to improve vision and when relating semester with areas affected with pain, and knowledge of the clinical posture of the dentist. A lower $\mathrm{p}<0.005$ value was found with respect to knowledge of clinical posture, being the VIII and $\mathrm{X}$ semesters, the ones with the less knowledge.

\section{Discussion}

Sim, Lacey and Lewis in 2006 established that there is a positive relationship between rigid postures and muscular skeletal disorders in different professions which could include pain, weakness, and paresthesia, this is widely documented and studied for a large number of professions. This study is in agreement with the results presented since a positive relationship was also found between muscular pain and inadequate postures in the subjects who participated in this study, but in contrast with the results of the authors already mentioned, this study only established this relationship for the dental profession (19).

Lindfors et al. in 2006 reported that the female group of dentists showed a higher incidence of muscular skeletal disorders; our study showed that the presence of pain was more frequent in females with $83 \%$ while in males was only $74 \%$.

Forced postures are frequently adopted by the dental students object of this study originating physiological alterations or muscular skeletal disorders which generate back and neck pain, among others. Valachi and Valachi in 2003 also reported these same symptoms but their study was done considering static postures for prolonged periods of time in dentists (1).

This study agrees with what was reported by Thornton, Stuart-Buttle, Wyszynski and Wilson in 2004, in their study "Physical and psychosocial stress exposures in US dental schools: the need for expanded ergonomics training", which estates that dental students adopt postures with high levels of muscular tension which are a possible risk factor for muscular skeletal disorders; in contrast with their study, our research did not evaluate the relationship between stress and muscular pain as did their study, which concluded that, besides adopting inadequate postures, the stress to which dental students are subjected, produces pain in the neck area, upper extremities, shoulders and lower spine (20).

\section{Conclusions}

Muscular pain is a common affliction in dentists which begins at the time they start their professional studies and it stays with them during their professional practice affecting the spine, neck, shoulders and hands, among others.

The results of this study show that adopting inadequate postures to gain better vision of the oral cavity, performing exaggerated flexions or cervical torsions could produce muscular pain. It was also concluded that there could be higher risk of muscular pain in clinical practice among the female population of this study, although no statistical significant differences were found and there was also a higher frequency of muscular pain in the approximating muscle of the thumb in both hands.

Adopting adequate postures in clinical practice and having a favorable work environment could reduce the frequency of lesions to the muscular skeletal system avoiding an early retirement from the profession. Therefore, it is of vital importance to promote occupational health training and prevention programs regarding ergonomic postures which must be acquired by the dental students during their clinical practices, originating in this manner, healthy lifestyles. 
It would be advisable to carry out cohort studies to establish with certainty, the cause-effect relationship of inadequate ergonomic postures during clinical dental practice and presence of muscular pain.

\section{References}

1. Valachi B, Valachi K. Preventing musculoskeletal disorders in clinical dentistry: strategies to address the mechanisms leading to musculoskeletal disorders. J Am Dent Assoc. 2003;134:1604-12.

2. Bramson JB, Smith S, Romagnoli G. Evaluating dental office ergonomic. Risk factors and hazards. J Am Dent Assoc. 1998;129:17483.

3. Caruso CC, Waters TR. A review of work schedule issues and musculoskeletal disorders with an emphasis on the healthcare sector. Ind Health. 2008;46:523-34.

4. Mandel ID. Occupational risks in dentistry: comforts and concerns. J Am Dent Assoc. 1993;124:40-9.

5. Armstrong TJ. Ergonomics and cumulative trauma disorders. Hand Clin. 1986;2:553-65.

6. Rising DW, Bennett BC, Hursh K, Plesh O. Reports of body pain in a dental student population. J Am Dent Assoc. 2005;136:81-6.

7. Alexopoulos EC, Stathi IC, Charizani F. Prevalence of musculoskeletal disorders in dentists. BMC Musculoskelet Disord. 2004;5:16.

8. Dong H, Loomer P, Barr A, Laroche C, Young E, Rempel D. The effect of tool handle shape on hand muscle load and pinch force in a simulated dental scaling task. Appl Ergon. 2007;38:525-31.

9. Bramson JB, Smith S, Romagnoli G. Evaluating dental office ergonomic. Risk factors and hazards. J Am Dent Assoc. 1998;129:17483.

10. Unthank M, True G. Interior design for dentistry. J Am Dent Assoc. 1999;130:1586-90.

11. Bare LC, Dundes L. Strategies for combating dental anxiety. J Dent Educ. 2004;68:1172-7.

12. Hyson JM Jr. The air turbine and hearing loss: are dentists at risk? J Am Dent Assoc. 2002;133:1639-42.

13. Park PR. Effects of sound on dentists. Dent Clin North Am. 1978;22:415-29.

14. Finkbeiner BL. Selecting equipment for the ergonomic fourhanded dental practice. J Contemp Dent Pract. 2001;2:44-52.

15. Sartorio F, Franchignoni F, Ferriero G, Vercelli S, Odescalchi L, Augusti D, et al. [Work-related musculoskeletal disorders in dentistry professionals. 2. Prevention, ergonomic strategies and therapeutic programs]. G Ital Med Lav Ergon. 2005;27:442-8.

16. Lindfors $P$, von Thiele U, Lundberg U. Work characteristics and upper extremity disorders in female dental health workers. J Occup Health. 2006;48:192-7.

17. Cagnie B, Danneels L, Van Tiggelen D, De Loose V, Cambier D. Individual and work related risk factors for neck pain among office workers: a cross sectional study. Eur Spine J. 2007;16:679-86.

18. Ebara T, Khuvasanont T, Krungkraiwong S, Amornratanapaichit R, Tachi N, Takeyama H, et al. Impact of ISO/TS 20646-1 "Ergonomic procedures for the improvement of local muscular workloads" on work-related musculoskeletal disorders. Ind Health. 2007;45:25667.

19. Sim J, Lacey RJ, Lewis M. The impact of workplace risk factors on the occurrence of neck and upper limb pain: a general population study. BMC Public Health. 2006;6:234.

20. Thornton LJ, Stuart-Buttle C, Wyszynski TC, Wilson ER. Physical and psychosocial stress exposures in US dental schools: the need for expanded ergonomics training. Appl Ergon. 2004;35:153-7. 